\title{
Segmental Duplications: A Possible Mechanism of Hominid Uplift through MicroRNA Diversification
}

\author{
Marla A. Endriga ${ }^{1}$, Aldrich Ivan Lois D. Burog${ }^{1}$, Denise Lauren V. Dalmacion ${ }^{1}$ and Custer C. Deocaris $^{2 *}$ \\ ${ }^{1}$ Department of Biology, College of Arts and Sciences, University of the Philippines Manila, 1000 Ermita, Manila, Philippines \\ ${ }^{2}$ Laboratory of Exercise Biochemistry, Institute of Health and Sports Sciences, University of Tsukuba, Japan
}

\begin{abstract}
MicroRNAs (miRNA) are $\sim 21$ nucleotide-long gene silencers. Segmental duplications (SD) are among the driving forces in acquiring new genes. Both miRNA and SD are believed to have played a significant role in evolution, particularly in the divergence of humans (Homo sapiens) from the chimpanzee (Pan troglodytes). This study determines the distribution of miRNAs in humans and in chimpanzees, and presents a hypothesis on its significance in the occurrence of segmental duplications. MiRNA sequences from miRBASE were subjected to BLAT and BLAST to determine if miRNAs are located in SD regions or not. Homology between miRNAs was determined with ClustalW. BLAST was then used to determine whether the non-homologous human miRNA are homologous to any other part of the chimpanzee genome. We found that all 695 human miRNAs are found exclusively in SD regions, and that 67 are de novo miRNAs. Thirteen are homologues of chimpanzee miRNA, and 11 were possibly derived from non-miRNA regions in chimp. Of these, 6 were located in SD regions of the chimpanzee genome. Results indicate that miRNA evolution occurs within regions of segmental duplication and suggest that the presence of miRNA duplicates allows more exposure to mutations that could necessitate diversification, and possibly evolution, through sub- and neofunctionalization.
\end{abstract}

Keywords:

\section{Introduction}

Genetic events such as gene translocations and mutations have been thought to be the sole driving force for evolution. However, recent studies have suggested that there exist other elements that contribute to the adaptive selection of a population through time. MicroRNAs and segmental duplication are genetic events that have gained much attention due to the new discoveries of their roles in disease manifestation, gene regulation and evolution (1-5).

The prevalence of segmental duplications or low copy repeats in the human genome characterizes and distinguishes humans from lower mammals. This provides an important insight on the potential role of segmental duplications in gene and genomic evolution (6). But the exact mechanism of evolution that

\section{*Corresponding address:}

Custer C. Deocaris, PhD,

Laboratory of Exercise Biochemistry, Institute of Health and Sports Sciences, University of Tsukuba, Japan

Email : cdeocaris@gmail.com allowed the human genome to diverge from that of the lower mammals to its present state remains uncertain.

Gene duplication results in the creation of novel gene function that could possibly drive the process of evolution (7). These duplicated genes are either lost or degraded into pseudogenes or retained through the course of evolution, depending on the selective advantage they confer on the organism $(8,9)$. The new gene duplicates acquire novel functions, though rarely, as they are driven by the force of adaptive evolution. They are subjected to a set of selective constraints that differs from that of the parental genes $(10,11)$. This is the mechanism of selection by which many gene functions come about from novel duplicated segments (12). This is true for a lot of primate-specific gene families (13-17). The ability to retain gene duplicates (or gene duplicability) increases the complexity of an organism derived from high adaptability (18). However, this duplicability varies among the eukaryotic gene families. Under the influence of gene regulation, it has been hypothesized that the primary factor that can explain the vast phenotypic divergence of humans and great apes are changes in regulation rather than having altered protein-coding gene sequences. Through comparative analysis, it has been shown that a 
high frequency of evolutionary conserved noncoding sequences exists in the vertebrate genome. These non-coding sequences might provide insight and contain the decisive alternatives for mode of regulation that may have motivated the course of human evolution (19).

This study determines the miRNA distribution in chimpanzees and humans as well as their occurrence within regions of segmental duplication, and proposes a hypothesis on their role in human evolution. This study adds new insights on the mode of miRNA diversification within regions of segmental duplication.

\section{Experimental Procedures}

Sources of miRNA sequences- The miRNA sequences of both human (Homo sapiens) and chimpanzee (Pan troglodytes) were acquired from the miRBase database (20) at http://microrna.sanger.ac.uk/sequences/index.s html. Stem-loop (precursor) sequences in FASTA format were utilized. As of September 2008, there were 595 chimp miRNA sequences, and as of March 2009, 695 human miRNA sequences were available.

Determination of miRNA distribution within segementally duplicated regions- A BLAT (21) search was done to determine if the human (695) and chimpanzee (595) miRNA sequences are located in SD regions or not. A miRNA sequence was considered if it met the following criteria: sequence identity of $100 \%$ with that of the query sequence derived from miRBase; and similar score and span size, with a span of at least $20 \mathrm{~kb}$. This size represents the minimum length of a mature or functional miRNA. The BLAT search for human miRNA sequences was applied to the March 2006 Assembly at the Centre for Applied Genomics Human Segmental Duplication Database (http://projects.tcag.ca/humandup/), while the BLAT search for nonhuman organisms, such as the chimpanzee, was done using the November 2003 genome assembly at the Centre for Applied Genomics Non-Human Segmental Duplication Database (http://projects.tcag.ca/xenodup/). Both are essentially databases of SD regions. The location of each miRNA -- chromosome number, strand, start and end bases - was noted.

Determination of miRNA in non-SD regions- Both human and chimpanzee miRNA sequences were also subjected to a BLAST (22) search at http://blast.ncbi.nlm.nih.gov/Blast.cgi to determine possible locations of miRNAs in non-
SD regions. The locations of the resulting miRNA sequences were compared with those in the BLAT search. This was done by comparing the coordinates of the miRNAs found using the two algorithms. This was done for both human and chimpanzee miRNA. Locations in BLAT that coincided with those in BLAST indicate that the corresponding miRNAs are restricted to $\mathrm{SD}$ regions. Locations in BLAT that are dissimilar with those in BLAST indicate that these miRNAs may be found in SD and in non$\mathrm{SD}$ regions.

MicroRNA Homology Search- Distinguishing nonhomologous from homologous miRNA sequences between human and chimpanzee was first achieved by comparing the miRNA IDs between chimp and human (http://microrna.sanger.ac.uk/sequences/). Based on the miRBASE nomenclature, similar numbers and letter suffixes of the ID between miRNA of different species indicate highly conserved or related (homologous) sequences. Human miRNAs with IDs not similar with any of the chimpanzee miRNA IDs were considered non-homologous (or de novo) miRNA.

Homologous human and chimpanzee miRNAs were subjected to multiple sequence alignment via ClustalW (available at http://www.ebi.ac.uk/Tools/clustalw/).

Pairwise scores (in percent) indicate the degree of similarity between sequences. This verifies if the sequences in the pair are identical and can be considered as homologous human-chimp miRNAs.

Each human miRNA that did not have a chimp miRNA counterpart was subjected to BLAST against the chimpanzee genome. This essentially aligns each human miRNA sequence against the entire chimpanzee genome and allows identification of sequences in the chimp genome (miRNA or non-miRNA) that are homologous to human miRNA. Human miRNAs with $100 \%$ identity, maximum of 4 mismatches, and zero gaps were considered homologous to the chimpanzee genome. Searches that generated no results were noted and considered as possible human miRNAs without chimp ancestry. To determine if those human miRNAs homologous to chimpanzee gene sequences are also chimpanzee miRNA, comparisons of BLAST chimpanzee miRNAchimpanzee genome alignments with BLAST human miRNA-chimpanzee genome alignments were done. These were further aligned with chimpanzee miRNAs using ClustalW to ensure 
Table 1. De novo human miRNAs resulting from chimpanzee BLAST search

\begin{tabular}{|c|c|c|c|c|c|}
\hline \multirow{2}{*}{$\begin{array}{c}\text { Accession } \\
\text { No. }\end{array}$} & \multirow[b]{2}{*}{ ID no. } & \multicolumn{2}{|l|}{ Accession } & \multicolumn{2}{|l|}{ Accession } \\
\hline & & No. & ID no. & No. & ID no. \\
\hline MI0000274 & hsa-mir-187 & MI0003653 & hsa-mir-638 & MI0006391 & hsa-mir-1257 \\
\hline MI0000299 & hsa-mir-222 & MI0003662 & hsa-mir-647 & MI0006395 & hsa-mir-548g \\
\hline MI0000342 & hsa-mir-200b & MI0003668 & hsa-mir-548d-1 & MI0006396 & hsa-mir-1261 \\
\hline MI0000449 & hsa-mir-132 & MI0003669 & hsa-mir-661 & MI0006400 & hsa-mir-548m \\
\hline MI0000476 & hsa-mir-138-1 & MI0003670 & hsa-mir-662 & MI0006402 & hsa-mir-548o \\
\hline MI0000486 & hsa-mir-190 & MI0003671 & hsa-mir-548d-2 & MI0006405 & hsa-mir-1268 \\
\hline MI0000764 & hsa-mir-363 & MI0003686 & hsa-mir-542 & MI0006406 & hsa-mir-1269 \\
\hline MI0000824 & hsa-mir-325 & MI0003763 & hsa-mir-767 & MI0006411 & hsa-mir-548h-1 \\
\hline MI0001641 & hsa-mir-429 & MI0003815 & hsa-mir-1301 & MI0006412 & hsa-mir-548h-2 \\
\hline MI0002467 & hsa-mir-483 & MI0003833 & hsa-mir-675b & MI0006413 & hsa-mir-548h-3 \\
\hline MI0003132 & hsa-mir-493 & MI0003834 & hsa-mir-769 & MI0006414 & hsa-mir-548h-4 \\
\hline MI0003560 & hsa-mir-92b & MI0005416 & hsa-mir-675 & MI0006419 & hsa-mir-1277 \\
\hline MI0003577 & hsa-mir-570 & MI0005537 & hsa-mir-888 & MI0006430 & hsa-mir-1283-2 \\
\hline MI0003581 & hsa-mir-574 & MI0006318 & hsa-mir-1228 & MI0006435 & hsa-mir-1255b- 1 \\
\hline MI0003592 & hsa-mir-585 & MI0006321 & hsa-mir-1231 & MI0006436 & hsa-mir-1255b-2 \\
\hline MI0003612 & hsa-mir-548a-3 & MI0006332 & hsa-mir-1200 & MI0006441 & hsa-mir-1308 \\
\hline MI0003615 & hsa-mir-602 & MI0006344 & hsa-mir-548e & MI0006652 & hsa-mir-1321 \\
\hline MI0003616 & hsa-mir-603 & MI0006347 & hsa-mir-1285-2 & MI0007075 & hsa-mir-1470 \\
\hline MI0003617 & hsa-mir-604 & MI0006371 & hsa-mir-1304 & MI0007259 & hsa-mir-1538 \\
\hline MI0003634 & hsa-mir-620 & MI0006376 & hsa-mir-548f-3 & MI0007260 & hsa-mir-1539 \\
\hline MI0003639 & hsa-mir-625 & MI0006377 & hsa-mir-548f-4 & MI0008330 & hsa-mir-1909 \\
\hline MI0003643 & hsa-mir-629 & MI0006378 & hsa-mir-548f-5 & MI0008335 & hsa-mir-1914 \\
\hline & & MI0006389 & hsa-mir-1255a & & \\
\hline
\end{tabular}

that sequences from the chimpanzee genome that will appear homologous to human miRNAs are also miRNAs (i.e. not coding regions of the genome). Searches yielding no similarity indicate that the human miRNA sequences are nonhomologous with the chimpanzee genome.

Human miRNAs found to be in the chimp genome but represent non-miRNAs in chimp were subjected to a BLAT search to identify possible locations of these human miRNA sequences in SD regions. Those query sequences (human miRNAs) with $100 \%$ identity and a similar query score and span were the criteria used to determine the presence of the query sequence in $\mathrm{SD}$ regions.

The methodology used in this study is summarized in Figure S.5.1 and Figure S.5.2.

\section{Results}

MicroRNA Distribution. The BLAT search revealed that out of the 595 of the chimpanzee miRNA, 564 are located in least one SD site. Twenty-three did not meet the criteria to be considered a miRNA sequence. No matches were found for two of the chimpanzee miRNA (ptr-mir-1281; ptr-mir-489).

On the other hand, of the 695 human miRNAs, all were found to be in SD regions. Locations for all 695 human miRNAs retrieved from BLAT coincided with those from BLAST.
Table 2. Human miRNAs with similarity to the chimp genome $($ BLAST score $=100 \%)$

\begin{tabular}{cc}
$\begin{array}{c}\text { Accession } \\
\text { number }\end{array}$ & ID no. \\
\hline MI0000650 & hsa-mir-200c \\
MI0000468 & hsa-mir-9-3 \\
MI0000093 & hsa-mir-92a-1 \\
MI0000112 & hsa-mir-105-2 \\
MI0000444 & hsa-mir-124-2 \\
MI0000455 & hsa-mir-138-2 \\
MI0000464 & hsa-mir-153-2 \\
MI0000488 & hsa-mir-194-1 \\
MI0003165 & hsa-mir-517b \\
MI0003182 & hsa-mir-519a-2 \\
MI0006346 & hsa-mir-1285-1 \\
MI0006429 & hsa-mir-1282 \\
MI0006657 & hsa-mir-1324 \\
MI0003677 & hsa-mir-655 \\
MI0006273 & hsa-mir-1180 \\
MI0006373 & hsa-mir-1243 \\
MI0007074 & hsa-mir-1469 \\
MI0006358 & hsa-mir-1297 \\
MI0007261 & hsa-mir-103-1-as \\
MI0007262 & hsa-mir-103-2-as \\
MI0003683 & hsa-mir-659 \\
MI0006379 & hsa-mir-1244 \\
MI0008333 & hsa-mir-1912 \\
MI0008336 & hsa-mir-1915 \\
\hline
\end{tabular}

MicroRNA Homology Search. Out of the 695 human miRNAs, 162 were not similar with any of the chimpanzee miRNA. BLAST alignment 
of these human miRNAs with the chimpanzee genome showed that 24 are conserved (0-4 mismatches, no gaps), while the other 67 had no similarities and hence, were considered human miRNAs without chimp ancestry (or de novo miRNAs). The latter are shown in Table 1 and the former are in Table 2.

BLAST results indicate that 13 out of the 24 human miRNAs have 100\% identity with known chimpanzee miRNAs and pairwise scores of $100 \%$, whereas the remaining 11 miRNAs showed $100 \%$ identity, but less than $100 \%$ pairwise scores in ClustalW. This means that 13 human miRNAs are identical to chimp miRNA, while 11 human miRNAs are present in chimpanzees, but not as miRNA. Table 3 shows the 13 human miRNAs with their respective chimpanzee miRNA counterpart identified with ClustalW alignments.

The chimpanzee BLAT search revealed that out of the 11 human miRNAs present in chimpanzee as non-miRNAs, 6 were in SD regions. All the hits from this search met the criteria for de novo miRNAs. Table 4 shows the summary of the query size, chromosome locations and scores that resulted from the search. Results of this study are summarized in Figure S.5.3.

Table 3. Human-chimpanzee miRNA pairs with pairwise score of $100 \%$

\begin{tabular}{|c|c|c|c|}
\hline \multicolumn{2}{|c|}{ Human miRNA } & \multicolumn{2}{|c|}{ Chimpanzee miRNA } \\
\hline Accession No. & ID no. & Accession No. & ID no. \\
\hline $\mathrm{MI0000093}$ & hsa-mir-92a-1 & $\mathrm{MI0003000}$ & ptr-mir-92-1 \\
\hline MI0000112 & hsa-mir-105-2 & MI0002749 & ptr-mir-105 \\
\hline MI0000444 & hsa-mir-124-2 & MI0002765 & ptr-mir-124a \\
\hline Ml0000455 & hsa-mir-138-2 & MI0008539 & ptr-mir-138 \\
\hline MI0000464 & hsa-mir-153-2 & MI0008553 & ptr-mir-153 \\
\hline MI0000488 & hsa-mir-194-1 & MI0003026 & ptr-mir-194 \\
\hline MI0003165 & hsa-mir-517b & MI0008711 & ptr-mir-517b-2 \\
\hline MI0003182 & hsa-mir-519a-2 & MI0008718 & ptr-mir-519a \\
\hline MI0006346 & hsa-mir-1285-1 & MI0008486 & ptr-mir-1285 \\
\hline MI0006429 & hsa-mir-1282 & MI0008481 & ptr-mir-1282-1 \\
\hline MI0006657 & hsa-mir-1324 & MI0008531 & ptr-mir-1324-1 \\
\hline & & MI0008532 & ptr-mir-1324-2 \\
\hline MI0006358 & hsa-mir-1297 & MI0008500 & ptr-mir-1297-1 \\
\hline MI0006379 & hsa-mir-1244 & MI0008441 & ptr-mir-1244-2 \\
\hline & & MI0008442 & ptr-mir-1244-3 \\
\hline & & MI0008444 & ptr-mir-1244-5 \\
\hline & & MI0008445 & ptr-mir-1244-6 \\
\hline & & MI0008447 & ptr-mir-1244-8 \\
\hline & & MI0008450 & ptr-mir-1244-11 \\
\hline
\end{tabular}

Table 4. BLAT Human miRNA with non-miRNA counterpart located in SD regions in chimpanzee genome.

\begin{tabular}{|c|c|c|c|c|c|c|c|c|c|c|}
\hline Query & Score & Start & End & QSize & Identity & Chromosome & Strand & Start & End & Span \\
\hline $\begin{array}{l}\text { hsa-mir- } \\
103-1 \text {-as } \\
\text { hsa-mir- }\end{array}$ & 62 & 1 & 62 & 62 & $100.00 \%$ & 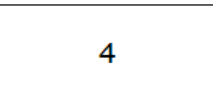 & + & 175165060 & 175165121 & 62 \\
\hline $\begin{array}{l}\text { 103-2-as } \\
\text { hsa-mir- }\end{array}$ & 62 & 1 & 62 & 62 & $100.00 \%$ & 21 & - & 3821998 & 3822059 & 62 \\
\hline $\begin{array}{l}1243 \\
\text { hsa-mir- }\end{array}$ & 93 & 1 & 93 & 93 & $100.00 \%$ & 3 & + & 131042991 & 131043083 & 93 \\
\hline $\begin{array}{l}1469 \\
\text { hsa-mir- }\end{array}$ & 47 & 1 & 47 & 47 & $100.00 \%$ & 16 & + & 95697059 & 95697105 & 47 \\
\hline $\begin{array}{l}1912 \\
\text { hsa-mir- }\end{array}$ & 80 & 1 & 80 & 80 & $100.00 \%$ & $\mathrm{X}$ & + & 117570186 & 117570265 & 80 \\
\hline 1915 & 80 & 1 & 80 & 80 & $100.00 \%$ & 8 & - & 22032997 & 22033076 & 80 \\
\hline
\end{tabular}

\section{Discussion}

It was discovered that the human miRNA sequences lie only in SD regions. With some exceptions, miRNA locations derived from the BLAT search of the Human Segmental Duplication Database were similar to those using BLAST and miRBase. For chimpanzees, on the other hand, BLAST-determined locations did not match those with BLAT. Not one result from BLAT showed similar coordinates with BLAST and miRBASE. This indicates that chimpanzee miRNAs are not found exclusively in $\mathrm{SD}$ regions.

What then is the significance of the preferential distribution of the human miRNAs in $\mathrm{SD}$ regions?
The exclusivity of human miRNAs in SD regions provides a major insight on the role of segmental duplications in the diversification of human miRNA. The duplicative transposition characteristic of segmental duplications probably provides a mechanism by which miRNAs can be derived from these transposable elements. The ubiquity, abundance and high evolutionary rate of transposable elements, such as those in segmental duplications, provide an impetus for miRNAs to emerge from such sequences. That the transposable elements are lineage-specific and mostly non-conserved suggests that miRNAs derived from such elements could also acquire the non-conserved trait that could provide diversifying regulatory effects on multiple genes. 
Segmental duplication events may have led to the production of de novo miRNAs, which in turn creates new functions (e.g. biochemical pathways, proteins, etc.). The presence of miRNAs in SD suggests that these miRNAs, through the course of evolution, may have been subjected to different modes of mutation and selection, which resulted in specialization or novel functions. It is possible that the presence of human miRNAs solely in SD regions conferred the selective advantages in terms of miRNA diversification. MiRNAs could have possibly acquired the opportunity in SD regions to diversify and be stably maintained in the genome, possibly through sub- or neofunctionalization. The interplay between conserved miRNAs and the miRNAs that are not of chimp ancestry might have contributed to increasing the functional complexity, allowing that certain organism to acquire new traits. And if these de novo miRNAs are species-specific, it would entail species-specific traits. Further, if these non-chimp human miRNAs have brainspecific targets, this could necessitate humanspecific traits, which could explain the differences between human and chimpanzee brains.

De novo miRNAs that are not of chimp ancestry were identified in this study. These might have played a role in the advancement of humans from the chimpanzees in molecular, anatomical and social aspects. Human miRNAs that were not found in any part of the chimpanzee genome might have particular gene targets in the human genome that corresponds to brain-specific functions. The presence of these miRNAs and their corresponding targets could provide a possible mechanism by which humans developed complex learning skills, language, enhanced memory and recall, and heightened capacity for interpretations, which led to the ability for more advanced cognitive tasks and develop social interactions and relationships with one another (23). Human miRNAs that were found in the chimp genome, but as non-miRNA, supports the idea that most miRNAs originate from non-miRNA regions, and that these may have been selected to become miRNAs depending on the environment (positive selection). It should be noted that a number of these human miRNA with counterparts in the chimpanzee genome are located in SD regions. This indicates that segmental duplication could be one of the mechanisms miRNAs evolve and possibly acquire new functions.

\section{Conclusion and Future Directions}

This study shows that human miRNAs are found exclusively in SD regions, while chimpanzee miRNA are not. Human miRNAs possibly derived from non-miRNA chimp gene sequences are present in humans, and some of these are found in SD regions in chimps. De novo miRNAs without chimp ancestry are also present in humans.

The presence of de novo miRNAs exclusively in human SD regions suggests that, over evolutionary time, these genes could have been subjected to numerous duplications. Some of these duplications could have provided sites for mutations to occur, particularly in miRNAs. Consequently, changes in miRNA could have resulted in the creation of functions specific to humans, for example, brain-specific ones. The fact that a number of human miRNAs are found in SD regions also indicates that segmental duplication could be one of the primary modes for miRNA evolution.

The preferential distribution of human miRNAs in segmentally duplicated regions supports the hypothesis that new miRNAs can be derived from transposable elements, particularly that of SD regions. However, we recommend that identification and annotation of the human miRNA gene targets be performed to determine brain-specific targets of both the homologous and de novo human miRNAs. Possible promoters and regulatory regions that might be adjacent to the novel miRNAs should be identified to determine their role in gene regulation and transcription. Network on the gene targets relevant to brain function should be performed to derive significant connections between brain-specific regulated genes.

\section{References}

[1] Eichler EE. Trends Genet. 2001; 17(11),661-9

[2] Pillai R S. RNA 2005; 11: 1753-1761

[3] Maher C, Stein L and Ware D. Genome Res 2006; 16:510-519

[4] Zhao Y and Srivastava D. Trends Biochem Sci 2007; 32(4):189-97

[5] Liu N, Okamura K, Tyler D, Phillips M, Chung W and Lai E. Cell Res 2008; 985-996

[6] Bailey J A, Gu Z, Clark RA, Reinert K, Samonte RV, Schwartz S, Adams MD, Myers EW, Li PW and Eichler EE. Science 2002; 297:1003-1007 
[7] Zhang J. Trends in Ecology and Evolution 2003; 18:292298

[8] Nadeau JH, Sankoff D. Genetics 1997; 147: 1259-1266

[9] Li WH, Gu Z, Wang H, Nekrutenko A. Nature 2001; 409: 847-849

[10] Walsh JB.Genetics 1995; 139: 421-428

[11] Lynch M, Conery JS.Science 2000; 290: 1151-1155

[12] Prince VE, Pickett FB.Nat Rev Genet 2002; 3: 827-837

[13] Courseaux A,Nahon JL.Science 2001; 291: 1293-1297

[14] Johnson ME, Viggiano L, Bailey JA, Abdul-Rauf M, Goodwin G, Rocchi M, Eichler E.E.Nature 2001; 413: 514-519

[15] Maston GA, Ruvolo M. Mol Biol Evol 2002; 19: 320335

[16] Zhang J, Zhang YP, Rosenberg HF. Nat Genet 2002; 30: $411-415$
[17] Paulding CA, Ruvolo M, Haber DA. Proc Natl Acad Sci 2003; 100:2507-2511

[18] Yang J, Lusk R, Li WH. Proc Natl Acad Sci 2003; 100:15661-15665

[19] Bird CP, Stranger BE, Liu M, Thomas DJ, Ingle CE, Beazley C, Miller W, Hurles ME, Dermitzakis ET. Genome Biol 2007; 8:R118

[20] Griffiths-Jones S. Nucleic Acids Res 2004; 32: D109D111 (http://microrna.sanger.ac.uk/sequences/ index.shtml.)

[21] Kent W J. (2002) Genome Res 12, 656-664

[22] Altschul SF, Gish W, Miller W, Myers EW, Lipman DJ. J Mol Biol 1990; 215 (3): 403-410.

[23] Bradbury J. PLoS Biol 2005; 3(3):e50 